\title{
TRANSPORT OF MHD COUPLE STRESS FLUID THROUGH PERISTALSIS IN A POROUS MEDIUM UNDER THE INFLUENCE OF HEAT TRANSFER AND SLIP EFFECTS
}

\author{
G.C. SANKAD* and P.S. NAGATHAN \\ Research Centre, Department of Mathematics \\ (Affiliated to Visvesvaraya Technological University, Belagavi) \\ B.L.D.E.A's V. P. Dr P.G.H CET, Vijayapur (586103) Karnataka, INDIA \\ E-mails: math.gurunath@bldeacet.ac.in; math.pratima@bldeacet.ac.in
}

\begin{abstract}
An attempt has been made to examine the effects of magnetohydrodynamic couple stress fluid in peristaltic flow with porous medium under the impact of slip, heat transfer and wall properties. The expressions are obtained for temperature, coefficient of heat transfer and velocity. Influences of different parameters, the Hartmann number, Brinkman number and adaptability parameters on the temperature and warmth trade coefficient are discussed through outlines.
\end{abstract}

Key words: peristaltic flow, couple stress fluid, Darcy number, slip parameter, compliant walls.

\section{Introduction}

The automatic contraction and relaxation of certain process in the human body induces progressive waves through which food and waste fluid move and is termed on peristalsis, for example, movement of chime in the gastrointestinal tract, urine flow through the ureter, blood flow through blood vessels. Peristaltic principle is also used by mechanical devices like roller and finger pumps. Beaver and Joseph [1] gave the first boundary condition for a permeable wall, verifying it theoretically and justifying statistically. Saffmann [2] improved this and suggested a condition suitable for small permeability. Studies on of peristaltic transport through a channel with compliant wall have been carried out by some researchers [3-7].

With a number of prospective applications in biomedical science, the study on peristalsis with heat transfer and MHD has attracted many researchers. The heat transfer concept is applied in computing the blood stream rate with the underlying thermal conditions. Bio-heat transfer is widely used in the destruction of unwanted tissues, laser therapy, cryosurgery, perfusion of blood stream, skin smoldering, fever and hypothermia in vertebrates. Peristalsis involving MHD is studied on account of its connection with movement of conductive physiological fluids, viz., stream of blood in small vessels and the pumping machines. The effect of wall characteristics on peristalsis involving heat transfer has been studied by Radhakrishnamacharya and Srinivasalu [8]. Hayat et al. [9] analyzed the importance of a compliant wall and heat transfer on the peristaltic flow through a curved channel considering an incompressible viscous fluid. The influence of heat transfer and wall properties on the peristaltic movement of power law fluid was observed by Hayat et al. [10].

The investigation of peristalsis through a permeable medium has gained importance in investigating the reflux conditions which is useful for realizing the intricacy of many diseases such as bacterial stones, bladder stones, cystitis, bacterial affection of kidneys, etc. Kothandapani and Srinivas [11] concentrated on the MHD peristaltic motion under heat transfer to analyze the wall property effect on the flow of a couple stress fluid. The incompressible laminar flow in a porous conduit of a couple stress fluid was analyzed by

\footnotetext{
* To whom correspondence should be addressed
} 
Srinivasacharya et al. [12]. Considering the food bolus movement through the esophagus as a Jeffrey fluid in a conduit, Arun et al. [13] examined the wall property and heat transfer effects. Dheia et al. [14] considered the flow through a porous medium channel in continuation of the work of [12]. The peristaltic flow of a Williamson fluid in an asymmetric channel with a porous medium was analyzed for studying the heat transfer and magnetic field effects by Ramesh and Devakar [15].

Assuming the arterial wall of the porous medium channel to be realistic, we have to consider the channel wall with slip condition as no slip condition is not valid at the wall due to permeability. Thus applying the slip condition means that the viscous effect is no longer present in the fluid. The study of flow through the channels with the slip is applied in chemical engineering, e.g., two phase flows in bearings of porous slides, flow through pipes in which reactions occur at the wall, etc. Srinivas et al. [16] considered the MHD peristaltic motion to investigate the slip wall property and also the heat transfer effect. Das [17] considered the collective effect of the slip wall, MHD and transfer of heat on the Maxwell fluid flowing through a peristaltic porous medium channel. The flow of a Newtonian fluid with couple stress inside a permeable medium channel was analyzed by Hummady and Abdulhadi [18] to study the effect of transfer of heat with slip conditions. The movement of an MHD Newtonian fluid in a permeable medium having flexible walls was investigated by Reddy and Reddy [19] to study the consequences of velocity slip and Joule heating. Recently, Laxshiminarayana et al. [20] examined the heat transfer effect of a conducting Bingham fluid in a non-uniform conduit. The peristaltic motion was considered for the study in the slip and elastic wall properties. It was concluded that with an increase in the magnetic parameter and the Brinkman number, there is a decrease in the temperature field, whereas the observed gain in the Brinkman number resulted in the rise of the heat transfer coefficient.

In the present study, the impact of heat transfer and wall slip condition on the peristaltic motion of a magnetohydrodynamic couple stress fluid is analyzed. The fluid is considered to flow through a uniform permeable flexible channel having a compliant wall. The flow has been investigated under the assumption that the: Reynolds number is small and wavelength of the peristaltic wave is large.

\section{Mathematical formulation}

The peristaltic transfer in a uniform channel between two elastic walls is considered as shown in Fig.1. The flow considered is unsteady, two dimensional and incompressible. The fluid in the channel is a magnetohydrodynamic couple stress fluid. Here dimensions of the uniform channel flow in a porous medium are given as the width of the uniform channel $2 d$, wave length $\lambda$ and wave amplitude $a . x$ is the axial coordinate and $y$ is normal to it.

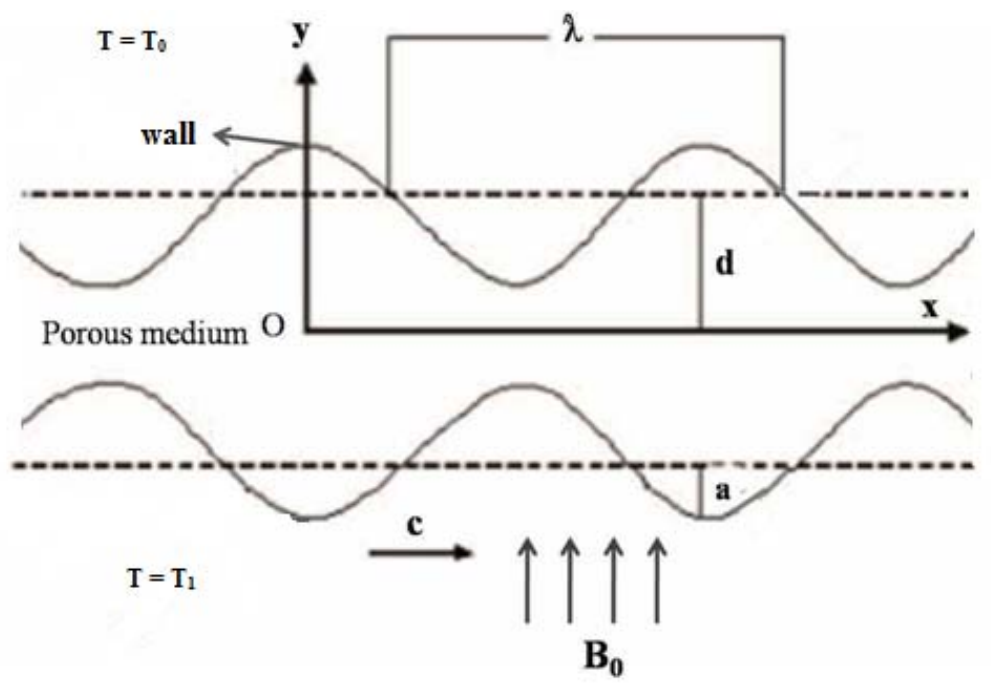

Fig.1. Physical model. 
The peristaltic wave is moving with constant speed $c$ along the channel wall and the geometry of the surface wall is

$$
y=\eta(x, t)=d+a \sin \frac{2 \pi}{\lambda}(x-c t) .
$$

The motion of the elastic wall is

$$
L(\eta)=p-p_{0}
$$

where $L$ is an operator that represents the flexible wall motion with viscosity damping force given by

$$
L=-\tau \frac{\partial^{3} \eta}{\partial x^{3}}+m \frac{\partial^{3} \eta}{\partial x \partial t^{2}}+C \frac{\partial^{2} \eta}{\partial x \partial t}
$$

Here the elastic tension in the wall is given by $\tau$, the mass per unit area is given by $m$ and the coefficient of the viscous damping force is given by $C$.

Basic equations are

$$
\begin{aligned}
& \frac{\partial u}{\partial x}+\frac{\partial v}{\partial y}=0 \\
& \rho\left(\frac{\partial u}{\partial t}+u \frac{\partial u}{\partial x}+v \frac{\partial u}{\partial y}\right)=-\frac{\partial p}{\partial x}+\mu \nabla^{2} u-h \nabla^{4} u-\mu \frac{u}{k}-\sigma B_{0}^{2} u \\
& \left(\frac{\partial v}{\partial t}+u \frac{\partial v}{\partial x}+v \frac{\partial v}{\partial y}\right)=-\frac{\partial p}{\partial y}+\mu \nabla^{2} v-h \nabla^{4} v-\mu \frac{v}{k} \\
& G\left(\frac{\partial T}{\partial t}+u \frac{\partial T}{\partial x}+v \frac{\partial T}{\partial y}\right)=\frac{K}{\rho}\left(\frac{\partial^{2} T}{\partial x^{2}}+\frac{\partial^{2} T}{\partial y^{2}}\right)+\vartheta\left\{2\left[\left(\frac{\partial u}{\partial x}\right)^{2}+\left(\frac{\partial v}{\partial y}\right)^{2}\right]+\left(\frac{\partial v}{\partial x}+\frac{\partial u}{\partial y}\right)^{2}\right\}
\end{aligned}
$$

In the equations above, the flow rates are $u$ and $v$ along $x$ and $y$ paths, respectively. As the plane is symmetrical, the normal velocity is zero. $k, \rho, \mu$ and $h$, respectively, represent the permeability of the porous medium, the fluid density, the coefficient of viscosity of the fluid and the constant associated with couple stress. Also, $p, \sigma, K, T, G$ and $\vartheta$ are the pressure, the electrical conductivity of the fluid, the thermal conductivity of the fluid, the temperature of the fluid, the specific heat at constant pressure and the kinematic coefficient of viscosity respectively. Further, $\nabla^{2}=\frac{\partial^{2}}{\partial x^{2}}+\frac{\partial^{2}}{\partial y^{2}}, \nabla^{4}=\nabla^{2} \nabla^{2}$. Hear we assume $p_{0}=0$.

The corresponding boundary conditions are

$$
\frac{\partial u}{\partial y}=0, \quad \frac{\partial^{3} u}{\partial y^{3}}=0, \quad \text { at } \quad y=0,
$$




$$
u=-d \frac{\sqrt{\mathrm{Da}}}{\beta} \frac{\partial u}{\partial y} \quad \text { at } \quad y= \pm \eta(x, t)
$$

where Da denotes the Darcy number and $\beta$ denotes the slip parameter.

After vanish the couple stresses

$$
\frac{\partial^{2} u}{\partial y^{2}}=0 \quad \text { at } \quad y= \pm \eta
$$

Referring to Mittra and Prasad [21] the dynamic boundary conditions are

$$
\frac{\partial}{\partial x} L(\eta)=-\rho\left(\frac{\partial u}{\partial t}+u \frac{\partial u}{\partial x}+v \frac{\partial u}{\partial y}\right)+\mu \nabla^{2} u-h \nabla^{4} u-\sigma B_{0}{ }^{2} u \quad \text { at } \quad y=\eta(x, t)
$$

where

$$
\frac{\partial}{\partial x} L(\eta)=\frac{\partial p}{\partial x}=-\tau \frac{\partial^{3} \eta}{\partial x^{3}}+m \frac{\partial^{3} \eta}{\partial x \partial t^{2}}+C \frac{\partial^{2} \eta}{\partial x \partial t}
$$

The non- dimensional quantities used are

$$
x^{\prime}=\frac{x}{\lambda}, \quad y^{\prime}=\frac{y}{d}, \quad t^{\prime}=\frac{c t}{\lambda}, \quad u^{\prime}=\frac{u}{c}, \quad v^{\prime}=\frac{\lambda v}{c d}, \quad p^{\prime}=\frac{d^{2} p}{\mu \lambda c}, \quad \eta^{\prime}=\frac{\eta}{d}, \quad \theta=\frac{T-T_{0}}{T_{1}-T_{0}} .
$$

The upper wall temperature is $T_{0}$ and at the lower wall the temperature is $T_{1}$.

Introducing non-dimensional variables in Eqs (2.4) - (2.11), after dropping the primes gives

$$
\begin{aligned}
& \frac{\partial u}{\partial x}+\frac{\partial v}{\partial y}=0, \\
& \operatorname{Re} \delta\left(\frac{\partial u}{\partial t}+u \frac{\partial u}{\partial x}+v \frac{\partial u}{\partial y}\right)=-\frac{\partial p}{\partial x}+\left(\delta^{2} \frac{\partial^{2} u}{\partial x^{2}}+\frac{\partial^{2} u}{\partial y^{2}}\right)+ \\
& -\frac{1}{\propto^{2}}\left(\delta^{2} \frac{\partial^{2}}{\partial x^{2}}+\frac{\partial^{2}}{\partial y^{2}}\right)\left(\delta^{2} \frac{\partial^{2} u}{\partial x^{2}}+\frac{\partial^{2} u}{\partial y^{2}}\right)-\frac{u}{k}-H_{0}^{2} u, \\
& \operatorname{Re} \delta^{3}\left(\frac{\partial v}{\partial t}+u \frac{\partial v}{\partial x}+v \frac{\partial v}{\partial y}\right)=-\frac{\partial p}{\partial y}+\delta^{2}\left(\delta^{2} \frac{\partial^{2} v}{\partial x^{2}}+\frac{\partial^{2} v}{\partial y^{2}}\right)+ \\
& -\frac{\delta^{2}}{\propto^{2}}\left(\delta^{2} \frac{\partial^{2}}{\partial x^{2}}+\frac{\partial^{2}}{\partial y^{2}}\right)\left(\delta^{2} \frac{\partial^{2} v}{\partial x^{2}}+\frac{\partial^{2} v}{\partial y^{2}}\right)-\delta^{2} \frac{v}{k},
\end{aligned}
$$




$$
\begin{aligned}
& \operatorname{Re} \delta\left(\frac{\partial \theta}{\partial t}+u \frac{\partial \theta}{\partial x}+v \frac{\partial \theta}{\partial y}\right)=\frac{1}{\operatorname{Pr}}\left(\delta^{2} \frac{\partial^{2} \theta}{\partial x^{2}}+\frac{\partial^{2} \theta}{\partial y^{2}}\right)+ \\
& +\operatorname{Ec}\left[2\left\{\delta^{2}\left(\frac{\partial u}{\partial x}\right)^{2}+\delta^{2}\left(\frac{\partial v}{\partial y}\right)^{2}\right\}+\left(\delta^{2} \frac{\partial v}{\partial x}+\frac{\partial u}{\partial y}\right)^{2}\right] .
\end{aligned}
$$

The relevant conditions at the boundary are

$$
\begin{aligned}
& \frac{\partial u}{\partial y}=0, \quad \frac{\partial^{3} u}{\partial y^{3}}=0 \quad \text { at } \quad y=0, \\
& u=-\frac{\sqrt{\mathrm{Da}}}{\beta} \frac{\partial u}{\partial y}, \quad \frac{\partial^{2} u}{\partial y^{2}}=0, \quad \text { at } \quad y= \pm \eta(x, t)= \pm(1+\varepsilon \sin 2 \pi(x-t)),
\end{aligned}
$$

and

$$
\begin{aligned}
& -\operatorname{Re} \delta\left(\frac{\partial u}{\partial t}+u \frac{\partial u}{\partial x}+v \frac{\partial u}{\partial y}\right)+\left(\delta^{2} \frac{\partial^{2} u}{\partial x^{2}}+\frac{\partial^{2} u}{\partial y^{2}}\right)-\frac{1}{\alpha^{2}}\left(\delta^{2} \frac{\partial^{2}}{\partial x^{2}}+\frac{\partial^{2}}{\partial y^{2}}\right)\left(\delta^{2} \frac{\partial^{2} u}{\partial x^{2}}+\frac{\partial^{2} u}{\partial y^{2}}\right)-H_{0}{ }^{2} u= \\
& =E_{1} \frac{\partial^{3} \eta}{\partial x^{3}}+E_{2} \frac{\partial^{3} \eta}{\partial x \partial t^{2}}+E_{3} \frac{\partial^{2} \eta}{\partial x \partial t} \quad \text { at } \quad y= \pm \eta(x, t) .
\end{aligned}
$$

Here, $\delta=\left(\frac{d}{\lambda}\right), \varepsilon=\left(\frac{a}{d}\right), \operatorname{Re}=\left(\frac{\rho c d}{\mu}\right), \mathrm{Da}=\frac{k}{d^{2}}, \propto=\left(\sqrt{\frac{\mu}{h}} d\right), H_{0}=B_{0} d \sqrt{\frac{\sigma}{\mu}}, \quad \operatorname{Pr}^{\prime} \frac{\rho \vartheta G}{K}, \quad \operatorname{Ec}=\frac{c^{2}}{G\left(T_{1}-T_{0}\right)}$, correspondingly stand for the wall slope parameter, the amplitude ratio, the Reynolds number, Darcy number, the couple stress parameter, the Hartmann number, the Prandtl number and the Eckert number.

The elasticity parameters are defined as $E_{1}=\left(\frac{-\tau d^{3}}{c \mu \lambda^{3}}\right), \quad E_{2}=\left(\frac{m c d^{3}}{\mu \lambda^{3}}\right), \quad E_{3}=\left(\frac{C d^{3}}{\mu \lambda^{2}}\right) . \quad$ The parameter $E_{1}$ represents the rigidity, $E_{2}$ - the stiffness and $E_{3}$ - the viscous damping force in the wall.

\section{Method of solving the problem}

Considering the wavelength of the peristaltic wave to be large and Reynolds number to be small, Eqs $(2.14)-(2.17)$ reduce to

$$
\begin{aligned}
& \frac{\partial u}{\partial x}+\frac{\partial v}{\partial y}=0, \\
& 0=-\frac{\partial p}{\partial x}+\frac{\partial^{2} u}{\partial y^{2}}-\frac{1}{\alpha^{2}} \frac{\partial^{4} u}{\partial y^{4}}-\frac{u}{k}-H_{0}{ }^{2} u
\end{aligned}
$$




$$
\begin{aligned}
& 0=-\frac{\partial p}{\partial y}, \\
& \operatorname{Ec}\left(\frac{\partial u}{\partial y}\right)^{2}+\frac{1}{\operatorname{Pr}} \frac{\partial^{2} \theta}{\partial y^{2}}=0 .
\end{aligned}
$$

The boundary conditions $(2.18)-(2.20)$ become

$$
\begin{aligned}
& \frac{\partial u}{\partial y}=0, \quad \frac{\partial^{3} u}{\partial y^{3}}=0, \quad \text { at } \quad y=0, \\
& u=-\frac{\sqrt{\mathrm{Da}}}{\beta} \frac{\partial u}{\partial y}, \quad \frac{\partial^{2} u}{\partial y^{2}}=0 \quad \text { at } \quad y= \pm \eta(x, t)= \pm(1+\varepsilon \sin 2 \pi(x-t)) \\
& \frac{\partial^{2} u}{\partial y^{2}}-\frac{1}{\alpha^{2}} \frac{\partial^{4} u}{\partial y^{4}}-H_{0}{ }^{2} u=E_{1} \frac{\partial^{3} \eta}{\partial x^{3}}+E_{2} \frac{\partial^{3} \eta}{\partial x \partial t^{2}}+E_{3} \frac{\partial^{2} \eta}{\partial x \partial t} \\
& \text { at } \quad y= \pm \eta(x, t) .
\end{aligned}
$$

Further

$$
\begin{aligned}
& \theta=0 \quad \text { on } \quad y=-\eta(x, t), \\
& \theta=1 \quad \text { on } \quad y=\eta(x, t) .
\end{aligned}
$$

The solution of Eqs (3.1) and (3.3), subject to the boundary conditions (3.5) - (3.7), is

$$
u=\frac{E}{M H 0^{2}}\left[\frac{m_{1} m_{2}-m 2^{2}}{H 0^{2} \propto^{2}}+\frac{m_{2}}{m_{1}}-1-M-\frac{m_{2} \cosh \left(\sqrt{m_{1}} y\right)}{m_{1} T_{1}}+\frac{\cosh \left(\sqrt{m_{2}} y\right)}{T_{2}}\right]
$$

Solving Eq.(3.4), with boundary conditions (3.8) and (3.9), we get

$$
\theta=P\left[\begin{array}{l}
\frac{m_{2}^{2}}{4 m_{1} T_{1}^{2}}\left\{\frac{\cosh 2 \sqrt{m_{1}} y}{2 m_{1}}-\frac{M 3}{2 m_{1}}-y^{2}+\eta^{2}\right\}+ \\
+\frac{m_{2}}{4 T_{2}^{2}}\left\{\frac{\cosh 2 \sqrt{m_{2}} y}{2 m_{2}}-\frac{M 4}{2 m_{2}}-y^{2}+\eta^{2}\right\}+ \\
-\frac{m_{2}{ }^{\frac{3}{2}}}{T_{1} T_{2} \sqrt{m_{1}}}\left\{\frac{\cosh M 1 y}{M 1^{2}}-\frac{\cosh M 2 y}{M 2^{2}}-\frac{M 5}{M 1^{2}}+\frac{M 6}{M 2^{2}}\right\}
\end{array}\right]+\frac{y}{2 \eta}+\frac{1}{2} .
$$

Here

$$
E=-\varepsilon\left[(2 \pi)^{3} \cos 2 \pi z\left(E_{1}+E_{2}\right)-E_{3}(2 \pi)^{2} \sin 2 \pi z\right],
$$




$$
\begin{aligned}
& M=\frac{m_{1} m_{2}-m_{2}^{2}}{H 0^{2} \propto^{2}}+\frac{\sqrt{\mathrm{Da}}}{\beta} m_{2}\left(\frac{T_{4}}{\sqrt{m_{2}}}-\frac{T_{3}}{\sqrt{m_{1}}}\right), \\
& H=\frac{1}{\mathrm{Da}}+H_{0}{ }^{2}, \quad m_{1}=\frac{\alpha^{2}+\alpha \sqrt{\alpha^{2}-4 H}}{2}, \quad m_{2}=\frac{\alpha^{2}-\alpha \sqrt{\alpha^{2}-4 H}}{2}, \\
& T_{1}=\cosh \sqrt{m_{1}} \eta, \quad T_{2}=\cosh \sqrt{m_{2}} \eta, \quad T_{3}=\operatorname{Tanh}\left(\sqrt{m_{1}} \eta\right), \\
& T_{4}=\tanh \left(\sqrt{m_{2}} \eta\right), \quad M 1=\sqrt{m_{1}}+\sqrt{m_{2}}, \quad M 2=\sqrt{m_{1}}-\sqrt{m_{2}}, \\
& M 3=\cosh 2 \sqrt{m_{1}} \eta, \quad M 4=\cosh 2 \sqrt{m_{2}} \eta, \quad M 5=\cosh M 1 \eta \\
& M 6=\operatorname{coshM} 2 \eta, \quad P=\frac{-\mathrm{Br} E^{2}}{M^{2} H 0^{4}}, \quad \text { and } \quad \mathrm{Br}=\mathrm{Ec} p_{r} \quad \text { is the Brinkman number. }
\end{aligned}
$$

The time average velocity $\bar{u}$ is

$$
\bar{u}=\int_{0}^{1} u d t .
$$

The heat transfer coefficient at the wall is

$$
\begin{aligned}
& H=\eta_{x} \theta_{y}(\eta) \\
& H=[2 \pi \varepsilon \cos 2 \pi(x-t)]\left[P \left\{\frac{m_{2}^{2}}{4 m_{1} T_{1}^{2}}\left(\frac{\sinh 2 \sqrt{m_{1}} \eta}{4 m_{1}^{3 / 2}}-2 \eta\right)+\frac{m_{2}}{4 T_{2}^{2}}\left(\frac{\sinh 2 \sqrt{m_{2}} \eta}{4 m_{2}^{3 / 2}}-2 \eta\right)+\right.\right. \\
& \left.\left.-\frac{m_{2}{ }^{\frac{3}{2}}}{T_{1} T_{2} \sqrt{m_{1}}}\left(\frac{\sinh M 1 \eta}{M 1^{3}}-\frac{\sinh M 2 \eta}{M 2^{3}}\right)\right\}+\frac{1}{2 \eta}\right] .
\end{aligned}
$$

\section{Numerical results and discussion}

The graphical results obtained from Eq.(3.11) are depicted in Figs 2-7 to show the effects of different parameters, i.e., the couple stress parameter $\alpha$, slip parameter $\beta$, Hartmann number $H_{0}$, Darcy number Da, Brinkman number $\mathrm{Br}$ and the effect of elastic parameters on temperature $\theta$ and heat transfer $H$. From Fig.2, we observe that the temperature decreases as the couple stress parameter increases. Figure 3 reveals that with the gain in the slip parameter $\beta$ there is a rise in the temperature. Looking at the effect of the Hartmann number $H_{0}$, from Fig.4, we a observe decrease in the temperature with an increase in $H_{0}$. Figure 5 shows an increase in the temperature with an increase in $\mathrm{Da}$. With a rise in $\mathrm{Br}$, the temperature also increases as shown in Fig.6. This result is in accordance with those obtained by Lakshminarayana et al. [20]. Figure 7 shows that by raising the elastic parameters, the temperature rises. 


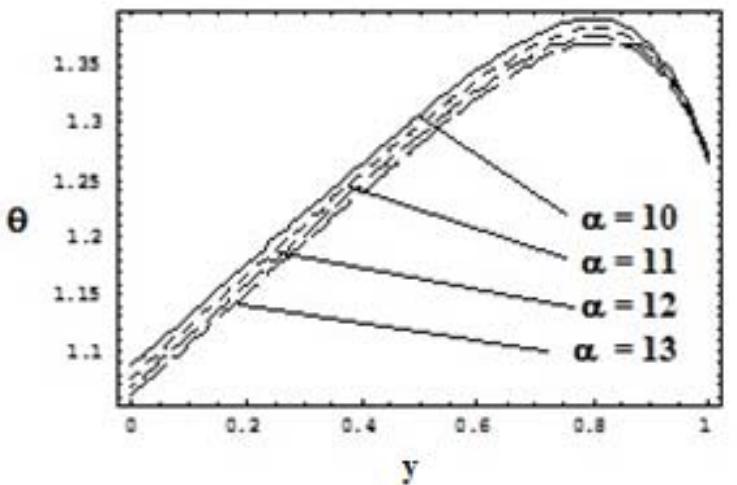

Fig..2. Plot of temperature.

$\varepsilon=0.2, E_{1}=0.1, E_{2}=0.5, E_{3}=0.3, \beta=1.1, \mathrm{Da}=0.1$,

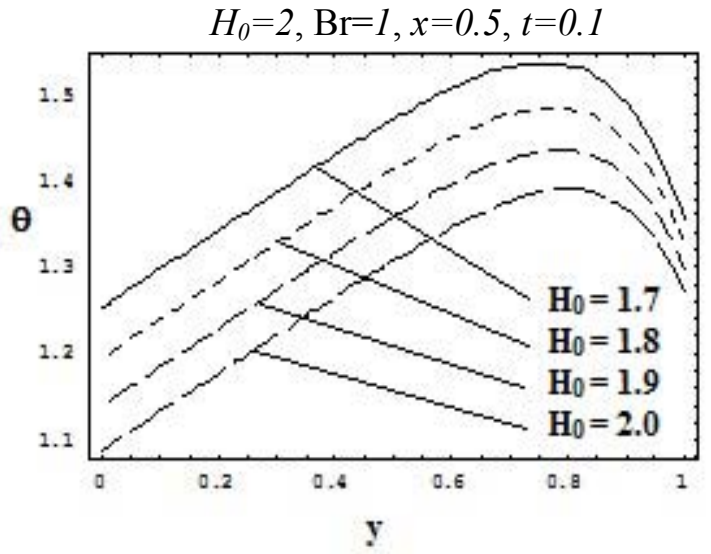

Fig.4. Plot of temperature.

$\varepsilon=0.2, E_{1}=0.1, E_{2}=0.5, E_{3}=0.3, \alpha=10, \beta=1.1$,

$\mathrm{Da}=0.1, \mathrm{Br}=1, x=0.5, t=0.1$

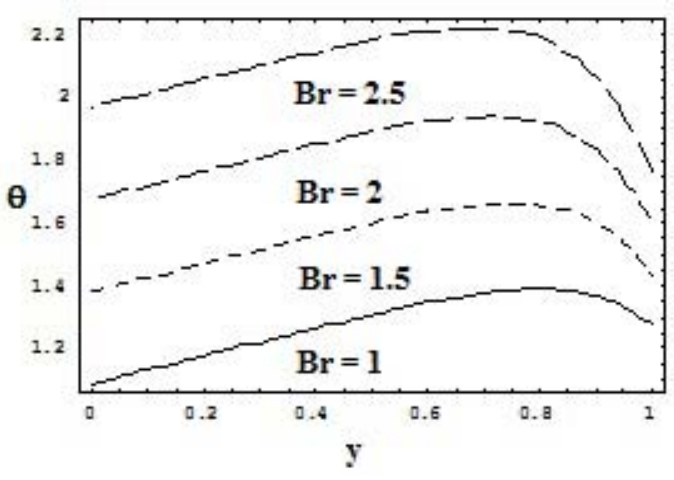

Fig.6. Plot of temperature.

$\varepsilon=0.2, E_{1}=0.1, E_{2}=0.5, E_{3}=0.3, \alpha=10, \beta=1.1$,

$H_{0}=2, \mathrm{Da}=0.1, x=0.5, t=0.1$

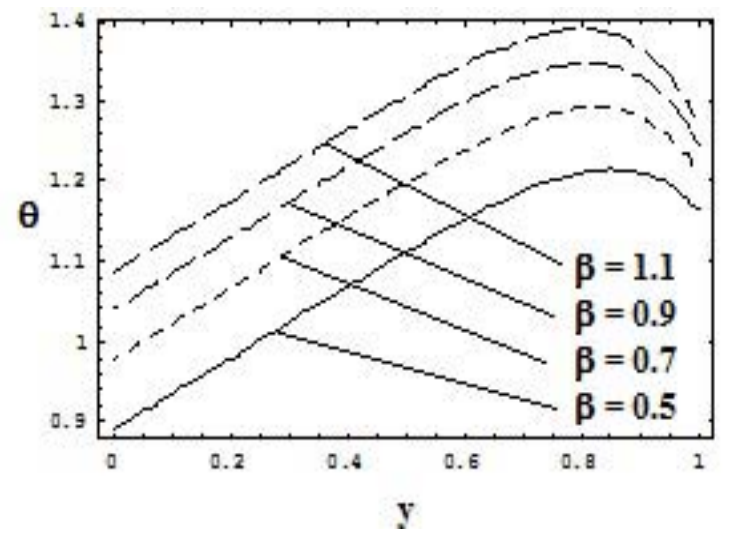

Fig.3. Plot of temperature. $\varepsilon=0.2, E_{1}=0.1, E_{2}=0.5, E_{3}=0.3, \alpha=10$,

$\mathrm{Da}=0.1, H_{0}=2, \mathrm{Br}=1, x=0.5, t=0.1$

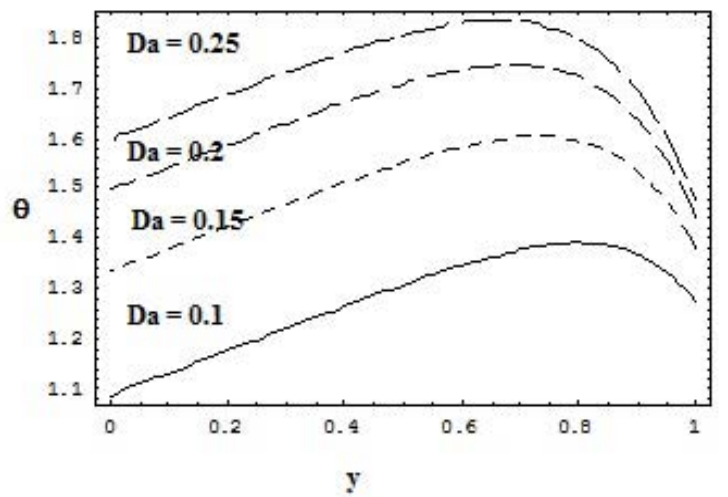

Fig.5. Plot of temperature.

$\varepsilon=0.2, E_{1}=0.1, E_{2}=0.5, E_{3}=0.3, \alpha=10, H_{0}=2$,

$\beta=1.1, \mathrm{Br}=1, x=0.5, t=0.1$

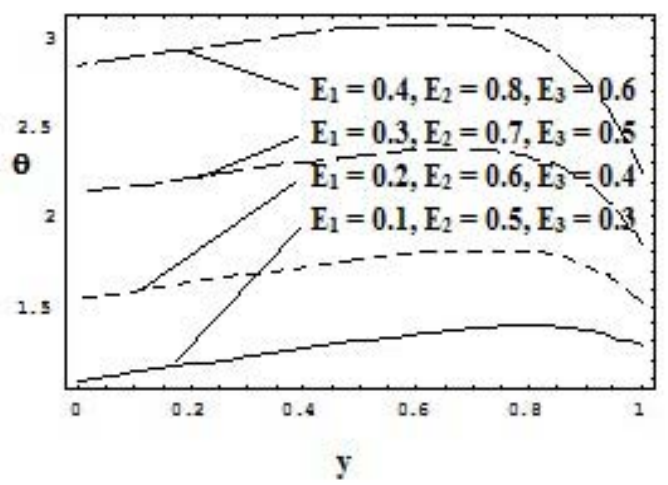

Fig.7. Plot of temperature.

$\varepsilon=0.2, \alpha=10, \beta=1.1, \mathrm{Da}=0.1, H_{0}=2, x=0.5$, $t=0.1$

A deviation of the heat transfer coefficient $H$ at the channel wall is shown in Figs 8-13 for different values of $\alpha, \beta, H_{0}, \mathrm{Da}, \mathrm{Br}, E_{1}, E_{2}$ and $E_{3}$ with preset values of the remaining parameters. These graphs 
present the absolute value of the coefficient of heat transfer. The absolute value of the coefficient of heat transfer $H$ reduces by enhancing $H_{0}$ and $\mathrm{Br}$ as depicted in Figs 8 and 9, whereas the magnitude of $H$ is increased by increasing the Darcy number Da as shown in Fig.10. The magnitude of the coefficient of heat transfer $H$ decreases by increasing elastic parameters $E_{1}, E_{2}$ and $E_{3}$ as shown in Fig.11. The effect of change in $\alpha$ and $\beta$ on $H$ is presented in Figs 12 and 13, but there is no appreciable difference.

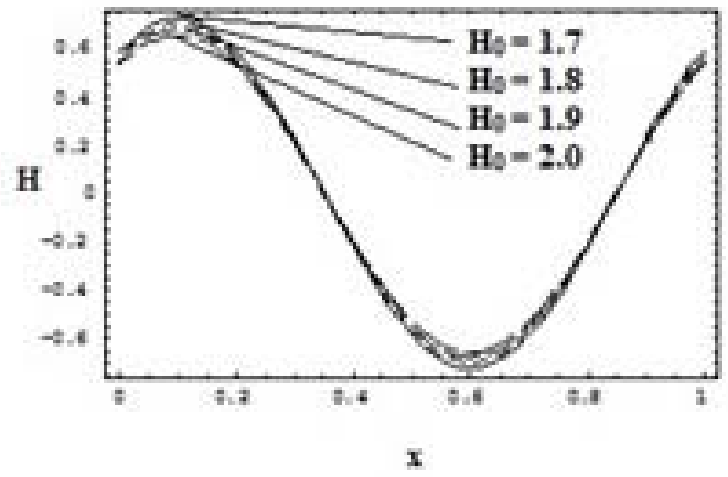

Fig.8. Plot of heat transfer coefficient. $\varepsilon=0.2, E_{1}=0.1, E_{2}=0.5, E_{3}=0.3, \alpha=10, \beta=1.1$, $\mathrm{Da}=0.1, \mathrm{Br}=1, x=0.5, t=0.1$

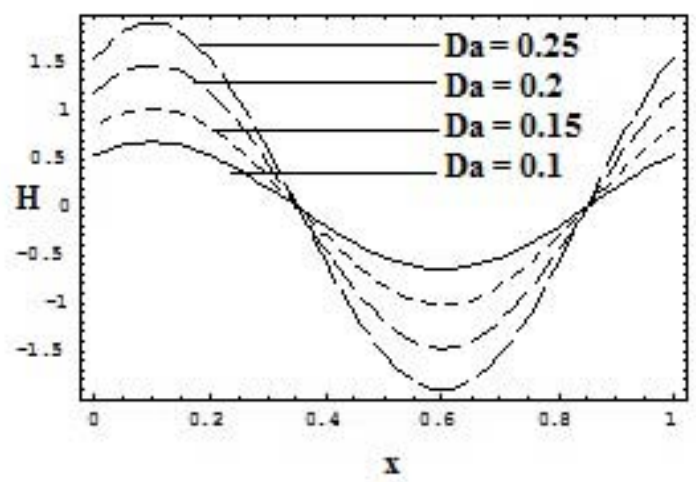

Fig.10. Plot of heat transfer coefficient. $\varepsilon=0.2, E_{1}=0.1, E_{2}=0.5, E_{3}=0.3, \alpha=10, H_{0}=2$, $\beta=1.1, \mathrm{Br}=1, x=0.5, t=0.1$

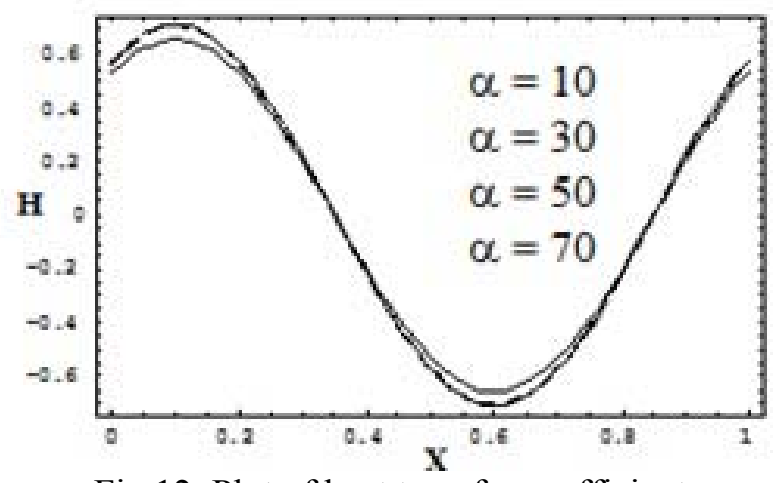

Fig.12. Plot of heat transfer coefficient. $\varepsilon=0.2, E_{1}=0.1, E_{2}=0.5, E_{3}=0.3, \beta=1.1, \mathrm{Da}=0.1$,

$$
H_{0}=2, \mathrm{Br}=1, x=0.5, t=0.1
$$

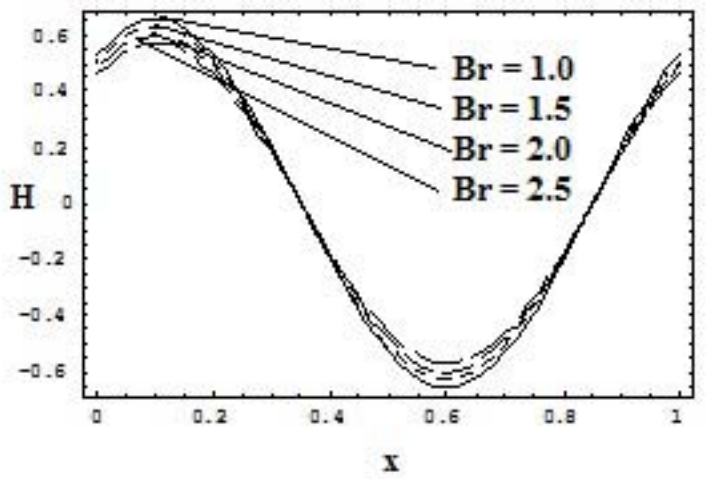

Fig.9. Plot of heat transfer coefficient. $\varepsilon=0.2, E_{1}=0.1, E_{2}=0.5, E_{3}=0.3, \alpha=10, \beta=1.1$, $H_{0}=2, \mathrm{Da}=0.1, x=0.5, t=0.1$

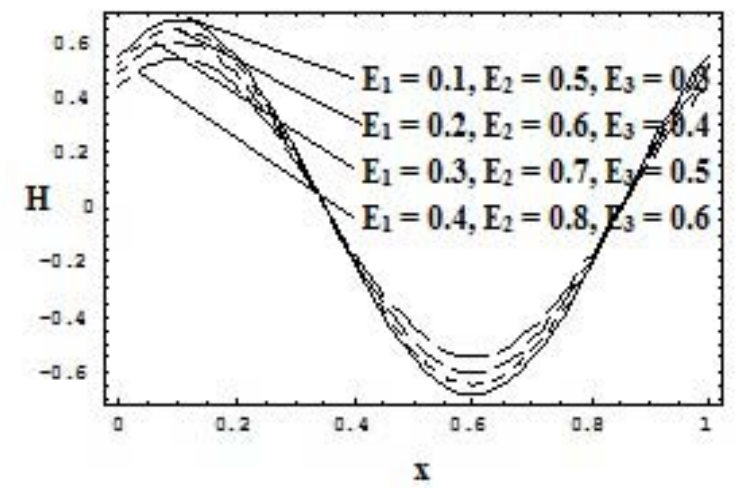

Fig.11. Plot of heat transfer coefficient. $\varepsilon=0.2, \alpha=10, H_{0}=2, \beta=1.1, \mathrm{Da}=0.1, x=0.5$, $t=0.1$

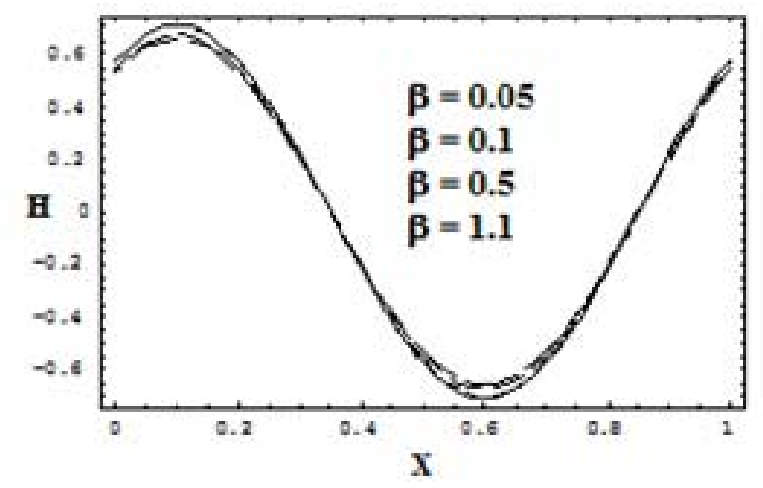

Fig.13. Plot of heat transfer coefficient. $\varepsilon=0.2, E_{1}=0.1, E_{2}=0.5, E_{3}=0.3, \alpha=10, \mathrm{Da}=0.1$, $H_{0}=2, \mathrm{Br}=1, x=0.5, t=0.1$ 


\section{Conclusion}

The motion of a magnetohydrodynamic couple stress fluid in a uniform channel with a porous medium under peristalsis is examined to study the heat transfer and slip effects. A theoretical solution is obtained using long wavelength and small Reynolds number assumptions. The consequences are analyzed for a variety of values of the parameters, namely; the couple stress parameter, Hartmann number, slip parameter, Brinkman number Darcy number and elastic parameters. The following interesting points have been noticed.

- Temperature increases with the increasing slip parameter, Darcy number, Brinkman number and also the elastic parameters.

- Temperature decreases with the increasing couple stress parameter and Hartmann number.

- The choice of the couple stress parameter depends on the Hartmann number while modelling peristaltic transport of physiological fluids together with the application of a magnetic field.

- In the upper part of the absolute value, the heat transfer coefficient increases with an increase in the Darcy number.

Our study on heat transfer is carried out having in view various applications, such as: destruction of cancer tissues, accessing skin burns, dilution method in investigating blood flow, food processing, etc.

\section{Nomenclature}

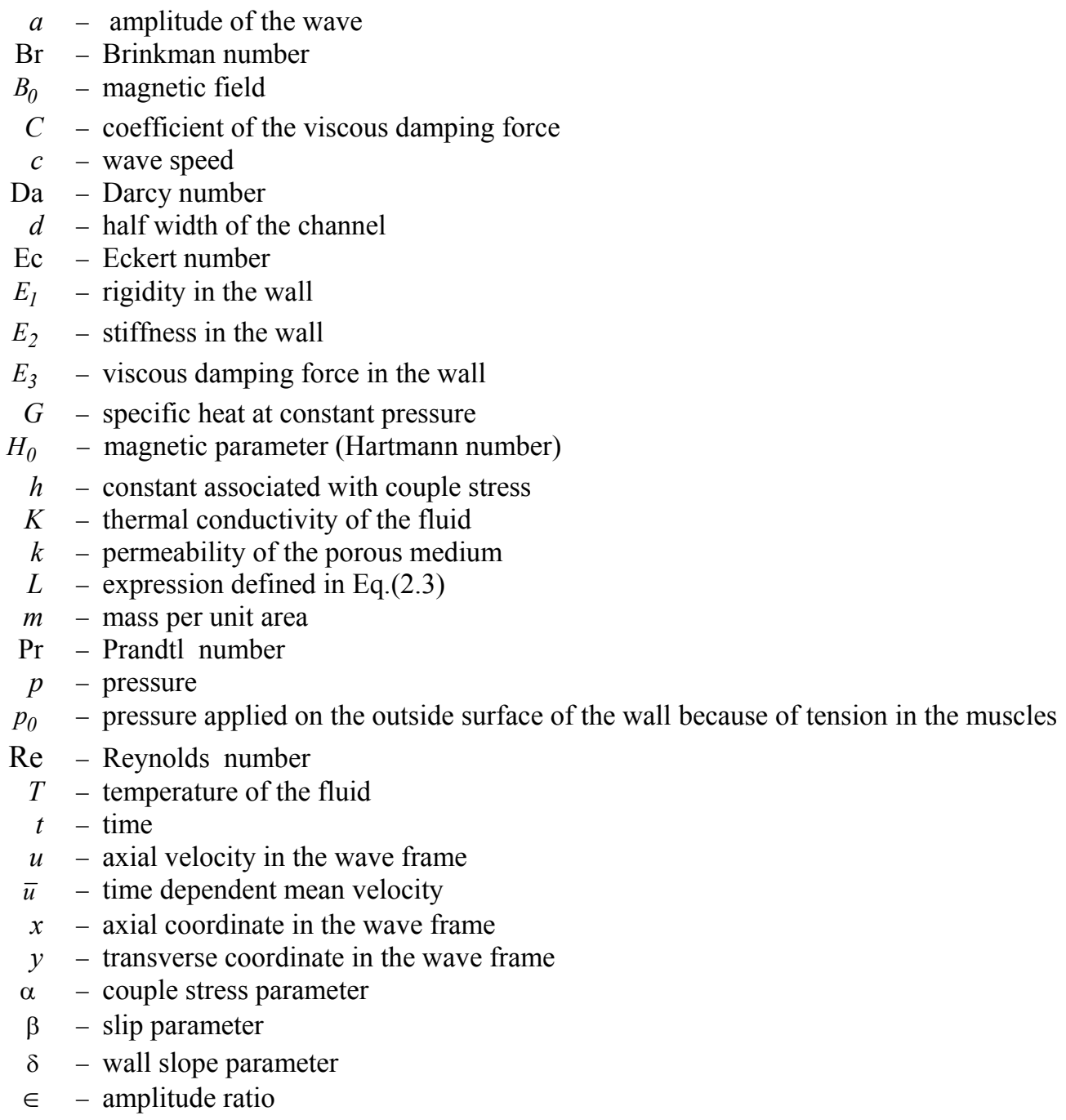


$\eta \quad$ - geometry of the wall defined in (2.1)

$\lambda$ - wavelength

$\mu \quad-$ coefficient of viscosity of the fluid

$v-$ transverse velocity in the wave frame

$\rho \quad-$ density

$\sigma \quad$ - electrical conductivity of the fluid

$\tau-$ elastic tension in the wall

$\vartheta \quad$ - kinematic coefficient

\section{References}

[1] Beaver G.S. and Joseph (1967): Boundary conditions magneto-fluid through a porous medium. - J. Fac. Educ. Ain Shams Univ. Egypt, vol.22, pp.35-51.

[2] Saffmann P.G. (1971): On the boundary condition at the surface of a porous medium. - Stud. Appl. Math, vol.1, pp.93-101.

[3] Abd Elnaby M.A. and Haroun M.H. (2008): A new model for studying the effect of wall properties on peristaltic transport of a viscous fluid. - Communications in Nonlinear Science and Numerical Simulation, vol.13, pp.752762.

[4] Ali N., Hayat T. and Asghar S. (2009): Peristaltic flow of a Maxwell fluid in a channel with compliant walls. Chaos, Solitons and Fractals, vol.39, pp.407-416.

[5] Hayat T. Javed M. and Ali N. (2008): MHD peristaltic transport of a Jaffrey fluid in a channel with compliant walls and porous space. - Trans. Porous Media, vol.74, pp.259-274.

[6] Parthasarathy S., Arunachalam G. and Vidhya M. (2015): Analysis on the effects of wall properties on MHD peristaltic flow of a dusty fluid through a porous medium. - International Journal of Pure and Applied Mathematics, vol.102, No.2, pp.247-263.

[7] Sankad G.C. and Radhakrishnamacharya G. (2011): Effects of magnetic field on the peristaltic transport of couple stress fluid in a channel with wall properties. - Int. J. Biomath, vol.4, No.3, pp.365-378.

[8] Radhakrishnamacharya G. and Srinivasulu C.H. (2007): Influence of wall properties on peristaltic transport with heat transfer. - Mecanique, vol.335, No.7, pp.369-373.

[9] Hayat T., Javed M., Awatif A. and Hendi A.A. (2011): Peristaltic transport of viscous fluid in a curved channel with compliant walls. - International Journal of Heat and Mass Transfer, vol.54, pp.1615-1621.

[10] Hayat T., Javed M., Asghar S. and Hendi A.A. (2012): Wall properties and heat transfer analysis of the peristaltic motion in a power-law fluid. - Int., vol.71, No.1, pp.65-79.

[11] Kothandapani M. and Srinivas S. (2008): On the influence of wall properties in the MHD peristaltic transport with heat transfer and porous medium. - Phys. Lett. A, vol.372, pp.4586-4591.

[12] Srinivasacharya N., Srinivasacharyulu and Odelu O. (2009): Flow and heat transfer of couple stress fluid in a porous channel with expanding and contracting walls. - International Communication in Heat and Mass Transfer, vol.36, No.2, pp.180-185.

[13] Arun Kumar M., Sreenadh S. and Srinivas A.N.S. (2013): Effects of wall properties and heat transfer on the peristaltic transport of a Jeffrey fluid in a channel. - Pelagia Research Library, Advanced in Applied Science Research, vol.4, No.6, pp.159-172.

[14] Dheia G. and Salih Al-Khafajy (2014): Effects of wall properties and heat transfer on the peristaltic transport of a Jeffrey fluid through porous medium channel. - Mathematical Theory and Modelling, vol.4, No.9, pp.86-99.

[15] Ramesh K. and Devakar M. (2015): Peristaltic transport of MHD Williamson fluid in an inclined asymmetric channel through porous medium with heat transfer. - J. Cent. South Univ., vol.22, pp.3189-3201.

[16] Srinivas S., Gayathri R. and Kothandapani M. (2009): The influence of slip conditions, wall properties and heat transfer on MHD peristaltic transport. - Computer Physics Communication, vol.180, pp.2115-2122. 
[17] Das K. (2012): Simultaneous effects of slip conditions and wall properties on MHD peristaltic flow of a Maxwell fluid with heat transfer. - Journal of Siberian Federal University, Mathematics and Physics, vol.5, No.3, pp.303315.

[18] Hummady Lika Z. and Abdulhadi A.M. (2014): Effect of heat transfer on the peristaltic transport of MHD with couple stress fluid through a porous medium with slip effect. - Mathematical Theory and Modelling, vol.4, No.7, pp.1-17.

[19] Venugopal Reddy K. and Gnaneswara Reddy (2014): Velocity slip and joule heating effects on MHD peristaltic flow in a porous medium. - Int. J. Adv. Math. and Mech., vol.2, No.2, pp.126-138.

[20] Lakshminarayana P., Sreenadh S. and Sucharitha G. (2015): The influence of slip, wall properties on the peristaltic transport of a conducting Bingham fluid with heat transfer. - Science Direct, Procedia Engineering, vol.127, pp.1087-1094.

[21] Mittra T.K. and Prasad S.N. (1973): On the influence of wall properties and poiseuille flow in peristalsis. - J. Biomech., vol.6, pp.681-693.

Received: June 30, 2016

Revised: March 10, 2017 